\title{
Balkanologie
}

Revue d'études pluridisciplinaires

Vol. $15 n^{\circ} 1 \mid 2020$

Mémoires performatives : faire des passés et des présents

\section{Mémoires mobilisées et compétitions mémorielles autour d'un espace sacré. La mise en héritage du Mont Athos (Grèce) depuis les années 1980}

Mobilized memories and memory competitions around a sacred space. The heritagization of Mount Athos (Greece) since the 1980s

Isabelle Dépret

\section{OpenEdition}

Journals

Édition électronique

URL : https://journals.openedition.org/balkanologie/2471

DOI : 10.4000/balkanologie.2471

ISSN : 1965-0582

Éditeur

Association française d'études sur les Balkans (Afebalk)

Référence électronique

Isabelle Dépret, « Mémoires mobilisées et compétitions mémorielles autour d'un espace sacré. La mise en héritage du Mont Athos (Grèce) depuis les années 1980 », Balkanologie [En ligne], Vol. 15 n 1 | 2020, mis en ligne le 01 juin 2020, consulté le 05 août 2021. URL : http://journals.openedition.org/ balkanologie/2471; DOI : https://doi.org/10.4000/balkanologie.2471

Ce document a été généré automatiquement le 5 août 2021.

(c) Tous droits réservés 


\section{Mémoires mobilisées et compétitions mémorielles autour d'un espace sacré. La mise en héritage du Mont Athos (Grèce) depuis les années 1980}

Mobilized memories and memory competitions around a sacred space. The heritagization of Mount Athos (Greece) since the 1980s

Isabelle Dépret

«Celui qui contrôle le passé (...) contrôle l'avenir.

Celui qui contrôle le présent contrôle le passé1.»

1 Haut lieu de la spiritualité et de l'imaginaire chrétiens orthodoxes, le Mont Athos, en tant qu'espace monastique, a traversé les époques byzantine et ottomane avant d'être intégré à l'État grec après la Première Guerre mondiale². Péninsule d'environ $335 \mathrm{~km}^{2}$ située aujourd'hui au nord de la Grèce, en Chalcidique, «l'État autonome de la Sainte Montagne" se structure autour de vingt grands monastères masculins, tous cénobitiques $^{3}$ à la fin $\mathrm{du} \mathrm{xx}^{\mathrm{e}}$ siècle, qui accueillent des religieux orthodoxes d'origines géographiques et linguistiques variées ${ }^{4}$. Les monastères athonites ne sont pas soumis à l'autorité de l'évêque local ni à celle des pouvoirs civils pour ce qui concerne leur administration interne ${ }^{5}$. Ce statut spécifique, ancré dans la longue durée ${ }^{6}$, est garanti par les constitutions grecques depuis $1926^{7}$.

2 Depuis les années 1980, alors que les politiques mémorielles et patrimoniales, jusqu'alors essentiellement stato-nationales sont redéfinies dans le cadre de l'Union européenne ${ }^{8}$, le Mont Athos se voit mis en valeur, voire revisité en terre d'héritage9. Dans un contexte de recomposition de l'Europe, l'inscription de la "Sainte montagne " sur la liste du patrimoine mondial de l'UNESCO en 1988 a représenté un jalon symbolique à cet égard ${ }^{10}$. 
3 Dans cet article, nous nous attacherons à saisir certains usages du passé, discours et pratiques mémorielles ayant accompagné ces processus. Nous nous appuierons sur des textes officiels, documents d'archives, publications médiatiques et données issues d'entretiens ou d'observations ${ }^{11}$ afin d'examiner les enjeux et les effets de ces mémoires mobilisées.

4 Si les mécanismes mémoriels restent, stricto sensu, des phénomènes individuels ${ }^{12}$, leurs composantes sociales ont été soulignées ${ }^{13}$, à tel point que les travaux consacrés aux "mémoires collectives ${ }^{14}$ » (leur mode de construction, leur possible instrumentalisation, leur rôle dans le déclenchement de conflits ou de réconciliations) se sont multipliés depuis les écrits du durkheimien Maurice Halbwachs dans les années $1920^{15}$. La notion, controversée, de mémoire «collective " renvoie à un ensemble de souvenirs, parfois issus d'expériences vécues mais le plus souvent transmis/appris, voire mythifiés, souvenirs censés être partagés par une communauté ${ }^{16}$.

5 Ces mémoires «collectives » étant bien difficiles à saisir, nous nous intéresserons surtout ici aux discours qui les évoquent et les mettent en scène, ainsi qu'aux actions commémoratives. Pour désigner cette mise en récit de la mémoire, qui peut déboucher sur des mémoires dominantes, officielles ou revendiquées, l'anthropologue Joël Candau utilise le terme de « métamémoire ${ }^{17}$ ».

\section{Un lieu de mémoire pour l'État-nation}

\section{Sélectionner et valoriser le passé national, axe majeur des politiques publiques grecques depuis le $\mathrm{XIX}^{\mathrm{e}}$ siècle}

6 Les historiens des $\mathrm{XIX}^{\mathrm{e}}-\mathrm{XX}^{\mathrm{e}}$ siècles se sont efforcés de bien séparer Histoire et mémoire : à une Histoire posée comme discipline scientifique, prétendant établir la vérité du passé par le biais d'une démarche rationnelle et rigoureuse ${ }^{18}$, s'opposeraient des mémoires, portées à gommer cette distance critique ${ }^{19}$, entretenant un rapport affectif et nécessairement sélectif aux événements révolus ${ }^{20}$.

7 L'antagonisme entre ces deux manières d'évoquer le passé au présent a été nuancé par les historiographes, les spécialistes d'histoire orale, les anthropologues et les psychologues sociaux des $\mathrm{xx}^{\mathrm{e}}-\mathrm{XXI}^{\mathrm{e}}$ siècles: la mémoire, $\mathrm{y}$ compris collective, se nourrirait en effet de l'histoire officielle et des travaux des historiens diffusés par l'entourage, l'institution scolaire et les mass media ${ }^{21}$. Résultats de l'évolution des mentalités, du regard des spécialistes et des demandes du politique, les histoires officielles d'hier peuvent être aujourd'hui analysées comme "récits ${ }^{22}$ " dont les liens avec le genre romanesque ne sont pas totalement coupés ${ }^{23}$. Enfin, les historiens, citoyens du présent, introduisent les préoccupations les plus contemporaines dans le choix de leur objet et dans la manière de le problématiser ${ }^{24}$.

8 Aussi, les usages de l'histoire par les États modernes afin de susciter, auprès des individus, des sentiments d'appartenance nationale, ont-elles fait l'objet de nombreux travaux depuis les années $1980^{25}$. Cette problématique est assurément pertinente pour étudier la construction des États-nations balkaniques/sud-est européens ${ }^{26}$, en l'occurence de la Grèce. 
Depuis la fondation du royaume de Grèce en $1830^{27}$, la mise en valeur (sélective) du passé a, en effet, toujours représenté un mode de légitimation pour l'État et une ressource en politique étrangère, culturelle et touristique. Exemple révélateur, le Service archéologique (1833) fut l'une des premières agences établies par l'État ${ }^{28}$. Au début du $\mathrm{xxI}^{\mathrm{e}}$ siècle, le ministère de la Culture hellénique apparaît encore, aux yeux de beaucoup, avant tout comme un « ministère de l'Archéologie ${ }^{29}$ ".

10 Si l'Antiquité grecque polythéiste a toujours occupé une place privilégiée pour les pouvoirs publics, dès la seconde moitié du XIX siècle, l'Empire byzantin et son héritage architectural, culturel, religieux se voient remis à l'honneur et érigés en seconde étape clé d'un récit national se voulant continu et plurimillénaire ${ }^{30}$ : perceptible dans la production historienne et la structure des manuels scolaires ${ }^{31}$, cette double priorité a eu des traductions concrètes, dont la fondation, en 1884, de la Société archéologique chrétienne puis, en 1914, du "Musée byzantin et chrétien », les deux adjectifs étant posés comme inséparables ${ }^{32}$. D'un point de vue institutionnel, ces choix politicoculturels font écho à la consolidation de rapports étroits entre l'Église orthodoxe et l'État ${ }^{33}$. A contrario, longtemps déniés sinon détruits, les vestiges trop « musulmans » de l'époque ottomane n'ont commencé à être envisagés comme éléments d'un patrimoine culturel local ou étatique que relativement récemment ${ }^{34}$.

$11 \mathrm{Au}$ tournant des $\mathrm{xx}^{\mathrm{e}}$-XXI $\mathrm{XI}^{\mathrm{e}}$ siècles, si l'éventail des domaines historiques institutionnellement promus en Grèce s'est étendu et si les problématiques de recherche se sont grandement renouvelées, l'empire chrétien oriental a conservé sa place de choix ${ }^{35}$. Le regard des pouvoirs publics sur le Mont Athos, emblème par excellence de cet héritage byzantin tant valorisé, prend son sens dans le cadre de ces représentations positives et du prestige accordé, en Grèce contempraine, aux études byzantines.

\section{Symphonia : catégorie de la mémoire collective en « terre orthodoxe » post-byzantine?}

12 Compte tenu des difficultés politico-financières de l'État grec et de l'attachement des moines à leur indépendance, la mise en valeur du patrimoine culturel et architectural athonite n'a pas constitué une priorité politique jusqu'aux années 1970-1980. Siégeant à Thessalonique, la $10^{\mathrm{e}}$ Éphorie des Antiquités byzantines ${ }^{36}$, service du ministère de la Culture compétent pour la Chalcidique et l'Athos, ne peut réaliser que des travaux fragmentaires au regard des besoins d'entretien d'un vaste ensemble. Il ne semble pas que les moines aient été, de leur côté, empressés d'ouvrir leurs «trésors » au monde extérieur ${ }^{37}$.

13 Le tournant a lieu au début des années 1980 lorsque la Grèce intègre l'Union européenne et que la sauvegarde du patrimoine est réaffirmée en valeur phare des missions étatiques, avec cette fois le soutien européen. En juillet 1981, le Parlement grec ratifie la Convention de l'Unesco de 1972 sur la « protection du patrimoine culturel et naturel ${ }^{38}$ ». À partir de ce moment, l'État hellénique mise sur la thématique patrimoniale ainsi que sur l'Europe - et ses financements - et engage un programme de travaux sur la « sainte Montagne ».

14 La candidature de l'Athos à l'Unesco sera portée par le gouvernement Andrea Papandreou (PASOK, parti socialiste) comme une cause nationale. Certes, pour ce parti, 
dont le programme pré-électoral de 1981 envisageait une séparation entre l'Église et l'État ${ }^{39}$, il s'agissait peut-être aussi de reconquérir les bonnes grâces du clergé orthodoxe, au moment même où était discutée, dans un climat des plus électriques, une loi visant à nationaliser une grande partie des propriétés rurales monastiques de Grèce ${ }^{40}$. Le Mont Athos et ses propriétés en Grèce étaient exclus du périmètre de cette $\operatorname{loi}^{41}$, qui ne fut, au demeurant, jamais appliquée ${ }^{42}$.

L'Unesco a posé dix critères de sélection au patrimoine mondial dans sa convention de 1972, parmi lesquels: «représenter un chef d'œuvre du génie créateur humain»; " apporter un témoignage (...) exceptionnel sur une tradition culturelle vivante ou disparue "; ou enfin "représenter des phénomènes naturels ou des aires d'une beauté exceptionnelle", "contenir les habitats naturels les plus représentatifs et (...) importants pour la conservation in situ de la diversité biologique $»^{43}$. Ici, la notion la plus classique du patrimoine culturel - le monument physique - s'est progressivement étendue tant aux traditions et éléments culturels "immatériels" qu'à l'idée selon laquelle la "nature ", dans sa diversité, représente aussi un " patrimoine » à protéger. Or, le Mont Athos tend à cumuler l'ensemble de ces critères : ensemble architectural et artistisque exceptionnel, traditions millénaires maintenues "vivantes", l'Athos est désormais aussi perçu comme un espace naturel particulièrement riche et beau et comme un îlot " écologique " précieux dans un monde en transformation rapide ${ }^{44}$. Pour justifier la candidature de l'Athos, le ministère grec met par ailleurs en avant les notions d'" intégrité » et d'" authenticité ", à l'honneur dans le lexique de l'Unesco ${ }^{45}$.

La promotion, par le ministère de la Culture hellénique, d'un «lieu de mémoire national » associé à l'héritage chrétien orthodoxe du pays venait conforter la position clé de la Grèce au sein de l'Europe culturelle. Mais ne réactivait-elle pas également, ce faisant, la logique de "Symphonia », cette tradition «byzantine» de soutien mutuel entre Église orthodoxe et État ${ }^{46}$ ?

La mise en héritage internationale de l'Athos à la fin $d u x^{e}$ siècle a ainsi pu être présentée comme une perpétuation de rapports traditionnels entre le pouvoir civil et les communautés monastiques protégées et bénéficiaires de privilèges: ainsi, les importantes subventions communautaires redistribuées par l'État grec ne font-elles pas écho aux anciennes chrysobulles impériales byzantines, pourvoyeuses de dotations et d'exemptions fiscales ${ }^{47}$ ?

\section{Les moines Hagiorites, dépositaires d'une tradition millénaire}

\section{Mémoires monastiques et tensions pour le contrôle du patrimoine de l'Athos}

18 Aux XX $^{e}$ et XXI ${ }^{e}$ siècles, pourtant, la « machine patrimoniale ${ }^{48}$ » tend à se distancier de la foi : les acteurs de l'État grec au $x^{e}$ siècle, généralement ouvertement croyants et grands admirateurs du monachisme athonite, ont certes oscillé entre une logique d'adhésion religieuse, notable par exemple dans le choix des gouverneurs civils du Mont Athos, et une logique, plus distanciée, de gestion «scientifique " d'un objet culturel et artistique. Les autorités civiles helléniques ont bien défendu le dossier de 
l'Athos auprès de l'Unesco : cela a-t-il signifié pour autant que les moines s'en sont réjouis?

$\mathrm{Si}$, en effet, depuis son passage sous souveraineté grecque, la péninsule athonite est bien un territoire ménagé par le pouvoir, en érigeant cet espace au rang d'« héritage ", l'État ne tend-il pas à le placer sous la tutelle des professionnels séculiers du patrimoine?

Déjà en 1965, lorsque l'État grec classait le Mont Athos en " monument à conserver ${ }^{49}$ ", les communautés athonites dénonçaient une «tentative de subordonner l'Athos à la juridiction et aux experts du ministère de la Culture ${ }^{50}$ ».

1 Dans une série de lettres et notes de la Sainte Communauté à l'État grec et à l'Unesco, les instances monastiques athonites fédérales se plaignent d'avoir été « ignorées » dans la procédure d'inscription de l'Athos au patrimoine mondial ${ }^{51}$. Selon ces mêmes instances, ce ne serait qu'après de « longs et douloureux efforts » que le ministère grec de la Culture aurait accepté d'associer pleinement les monastères à l'entreprise. Depuis le début des années 2000, les monastères, en tant qu'organismes de droit public, sont ainsi habilités à prétendre directement aux financements européens, sans passer par les rouages administratifs de l'État hellénique ${ }^{52}$.

Il est révélateur que, lors de plusieurs séminaires parrainés par l'Unesco au début des années 2000, les représentants des monastères soulignent à quel point les «questions de patrimoine religieux, culturel et naturel » relèveraient "par excellence (...) de la compétence de la Sainte Communauté athonite elle-même» et non d'un pouvoir " extérieur " ${ }^{53}$; et que seul le maintien des règles traditionnelles, marquées par la noningérence du pouvoir séculier dans les affaires de l'Athos, permettrait de préserver, à la fois pure et vivante, la « mémoire monastique » hagiorite ${ }^{54}$.

\section{Reproduction et usages de la tradition hagiorite}

3 Finalement, à la fin $\mathrm{du} \mathrm{xx}^{\mathrm{e}}$ siècle, la mémoire nationale en Grèce représente une référence polysémique, conflictuelle sur certains épisodes ${ }^{55}$, tandis que d'autres ont été repoussés, doucement ou violemment, dans l'oubli ${ }^{56}$. plutôt sécularisée, valorisant la "résistance » et les «combats du peuple ${ }^{57}$ ». Elle ne s'ajuste que partiellement aux logiques et pratiques mémorielles monastiques, centrées sur le culte des saints et des ancêtres ${ }^{58}$, sur le cycle des commémorations religieuses, sur l'attachement à des traditions et à des textes remontant parfois à l'époque paléochrétienne, byzantine ou ottomane ${ }^{59}$ et, enfin, sur la défense farouche d'une autogestion pour la communauté religieuse, autogestion devenue, en ce sens, un paramètre de l'identité, et de la mémoire, monastique sur l'Athos ${ }^{60}$.

Les textes publiés par des religieux et les articles internet consacrés à l'Athos tendent à souligner à quel point le monachisme athonite perpétuerait, souvent à l'identique depuis l'époque byzantine, une tradition millénaire, une spiritualité et un mode de vie fait de prière, d'ascèse, de travaux manuels ou de lectures ${ }^{61}$. Dans nombre de ces textes, les moines athonites semblent "naturalisés", posés comme les monuments vivants d'un paysage physique et moral et d'un passé immuable qu'ils contribueraient activement à sauvegarder ${ }^{62}$. 
26 Et pourtant, au-delà de la notion capitale de fraternité universelle ou orthodoxe, les mémoires monastiques restent plurielles. La tradition orthodoxe, mémoire certes partagée, peut être invoquée pour justifier des attitudes variables. Au tournant des $\mathrm{xx}^{\mathrm{e}}$ et $\mathrm{xxI}^{\mathrm{e}}$ siècles, par exemple, deux monastères athonites voisins, Vatopaidi et Esphigmenou, privilégient des lectures et usages un peu différents de la tradition ${ }^{63}$.

Probablement fondé aux $\mathrm{x}^{\mathrm{e}}$ et $\mathrm{xI}^{\mathrm{e}}$ siècles de notre ère, le monastère d'Esphigmenou est habité dans les dernières décennies du $\mathrm{xx}^{\mathrm{e}}$ siècle par une communauté zélote, attachée à l'ancien calendrier julien ${ }^{64}$. Ces moines, hostiles à toute idée de « changement » ou de "modernisation», notions identifiées à autant de corruptions d'une tradition à préserver « intacte », vivaient encore au début des années 2000 dans des conditions de vie rudimentaires. Devenue célèbre pour ses grands drapeaux noirs sur lesquels était inscrit : «L'Orthodoxie ou la mort », la communauté zélote d'Esphigmenou a refusé les financements européens, considérés comme un cadeau empoisonné : une stratégie de subordination de l'Athos à l'Europe occidentale "papiste » ${ }^{65}$. Les moines d'Esphigmenou, déclarés «schismatiques " par la Sainte Communauté en $2002^{66}$, ont rompu depuis les années 1970 avec leur autorité spirituelle, le Patriarcat de Constantinople, coupable selon eux de trahison et de rapprochement avec l'ennemi, l'Église catholique de Rome ${ }^{67}$.

Établi à la fin du $\mathrm{x}^{\mathrm{e}}$ siècle, le monastère de Vatopaidi ${ }^{68}$ est aujourd'hui l'un des plus prospères de l'Athos, les moines y résidant étant toutefois des ascètes ${ }^{69}$. Au début du $\mathrm{xxI}^{\mathrm{e}}$ siècle, il compte plus de cent moines, dont beaucoup sont originaires de Chypre. Une grande partie d'entre eux a moins de 40 ans ; la moitié est également diplômée de l'enseignement supérieur et largement insérée dans la civilisation internet ${ }^{70}$. Ce monastère s'est montré plus souple et plus prompt à intégrer certains éléments de la "modernité », notamment de nouvelles technologies de communication ou de gestion, afin de promouvoir un idéal religieux, voire le monastère lui-même. Les moines produisent et commercialisent aussi quelques produits (vin, huile, olives, épices...) dans le respect des «traditions ancestrales ». Le monastère de Vatopaidi recèle, par ailleurs, une série de reliques, dont un morceau de la Vraie Croix, la Ceinture de la Vierge Marie et le Crâne de St Jean Chrysostome, et possède au moins huit icônes de la Vierge.

\section{Une arène mémorielle internationale : commémoration et influence politico-nationale sur l'Athos au début du $\mathrm{xxI}{ }^{\mathrm{e}}$ siècle}

29 À la fin des années 1980, la Grèce est encore le seul État de tradition chrétienne orthodoxe membre de la communauté européenne. Cette position a tôt été conçue comme un atout face à d'autres États culturellement représentés sur le Mont Athos : Bulgarie, Serbie, Monténégro, Roumanie, Russie... Les programmes intégrés européens constituent la source d'importantes subventions ${ }^{71}$. Une portion de ces fonds - au total plusieurs dizaines de millions d'euros - a permis, depuis les années 1980-1990, la réalisation de travaux de restauration des établissements athonites ${ }^{72}$. Une part majeure des actions de protection et d'aménagement réalisées depuis les années 1980 est liée aux financements européens.

30 Ce souci de marquer la claire souveraineté grecque sur l'Athos est renforcé après l'effrondrement du bloc soviétique : durant les années 1990, le renouveau religieux en 
Europe orientale et la profonde crise morale et économique de ces sociétés suscitent l'arrivée de novices et de moines issus des Balkans et de l'ancienne Union soviétique. Cet afflux aurait alors été perçu par des cadres du ministère grec des Affaires étrangères et par certains monastères comme un risque d'altération de la "composition ethnique» de l'Athos. L'ouvrage publié par la Sainte Communauté athonite en 1996 - Le régime du Mont Athos - témoigne tout spécialement de ces préoccupations ; une décision du Conseil d'État, en 1993, rappelle que l'installation d'un moine sur l'Athos doit être déclarée et contrôlée par le pouvoir civil étatique ${ }^{73}$.

Dans Une nation pour mémoire, ouvrage centré sur la France, l'historien Pascal Ory rattache les cérémonies commémoratives aux "politiques symboliques»: les remémorations publiques d'événements historiques comportant ici une dimension participative, émotionnelle et festive, sont autant de pratiques pouvant avoir une "efficacité » dans la cristallisation d'une «mémoire collective ${ }^{74}$ ». L'auteur, qui envisage différents "procédés commémoratifs", distingue l'exposé (reposant sur un discours, une narration) de l'exposition (reposant sur le visuel: montrer un site, un monument, une collection d'objets, un spectacle ${ }^{75}$ ). Nous reprenons cette distinction pour mettre en exergue une surenchère mémorielle sur l'Athos au tournant des $\mathrm{xx}^{\mathrm{e}}$ et $\mathrm{xxI}^{\mathrm{e}}$ siècles, reflet d'une subtile compétition politique entre la Grèce, associée à l'Union européenne, et la Russie.

\section{L'exposition « Les Trésors du Mont Athos » (1997). Les projets de rayonnement de Thessalonique, arrière-cour de l'Athos}

Dans les années 1990, la Grèce du Nord est travaillée par un double mouvement : d'une part, la peur et les crispations nationalistes, réactions aux destabilisations qui accompagnent la chute du bloc soviéto-communiste en Europe balkanique; d'autre part, la perception accrue du potentiel de rayonnement d'une région, la Macédoine grecque, et d'une ville ancienne, Thessalonique ${ }^{76}$, "réduite» au rang de seconde capitale provinciale depuis les années $1920^{77}$. Les années 1990, marquées à Thessalonique par de grandes manifestations patriotiques pour défendre le « caractère hellénique » de la Macédoine et par le maintien au pouvoir du camp conservateur ${ }^{78}$, sont aussi celles de projets de développement urbain, grâce aux financements étatiques et européens ${ }^{79}$. En 1997, Thessalonique est sélectionnée comme «capitale européenne de la culture ${ }^{80} »$.

33 C'est dans ce cadre qu'est organisée, du 21 juin au 31 décembre 1997, l'exposition « Les trésors du Mont Athos» qui présente 1500 objets témoins d'une «histoire millénaire ${ }^{81}$ ». Inaugurée par le ministre de la Culture Evangelos Venizelos (PASOK), l'exposition, préparée par l'organisme "Thessalonique, capitale culturelle de l'Europe ", la municipalité et le musée byzantin de la ville, a impliqué la collaboration de 17 monastères athonites, ayant accepté, non sans hésitations, de prêter des pièces conservées dans leurs murs ${ }^{82}$.

Dans le sillage de cette exposition, qui fut une "réussite " eu égard au nombre considérable de visiteurs ayant afflué, est institué en 2000 le Centre du Mont Athos

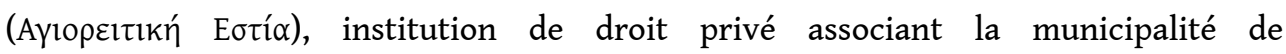
Thessalonique et la Sainte Communauté de l'Athos autour d'un objectif commun: promouvoir le Mont Athos et mieux faire connaître son «rayonnement spirituel, culturel » passé et présent ${ }^{83}$. 
35 À la fin des années 1990, en soulignant des liens traditionnellement privilégiés avec le Mont Athos, les élites municipales, avec l'appui de l'échelon étatique grec, tendent à revaloriser Thessalonique comme métropole balkanique et européenne, symbole d'un héritage chrétien orthodoxe et byzantin, qui réémerge comme facette, moins connue, de l'histoire européenne ${ }^{84}$. Parallèlement, les higoumènes de plusieurs monastères athonites s'ouvrent, non sans réticences, à l'Union européenne, dont les programmes de préservation patrimoniale permettent des financements jugés utiles.

C'est dans ce contexte qu'il faut replacer la politique religieuse et mémorielle de l'État russe autour de l'Athos au tournant des $\mathrm{xx}^{\mathrm{e}}$ et $\mathrm{xxI} \mathrm{e}^{\mathrm{e}}$ siècles.

\section{La commémoration des mille ans de présence russe sur l'Athos}

Dévaluée à l'époque de l'Union soviétique et de la Guerre froide, la politique «religieuse » étrangère de la Russie, ancrée dans une longue tradition impériale et structurée autour du patronage de l'orthodoxie, se trouve revitalisée au début du $\mathrm{xxI}^{\mathrm{e}}$ siècle, dans un contexte de tensions renouvelées avec les États-Unis et avec l'Union européenne.

La remise en avant de la religion orthodoxe comme outil de politique internationale se confirme après l'arrivée au pouvoir, en 1999, de Vladimir Poutine ${ }^{85}$, qui se présente comme un fervent croyant $^{86}$. Le Mont Athos qui, comme le rappelle le Patriarcat de Moscou, comptait 1446 moines russes en $1903^{87}$, soit plus de la moitié de la population monastique athonite d'alors, a été l'un des théâtres de la politique culturelle et religieuse russe en Europe au début du $\mathrm{xxI}^{\mathrm{e}}$ siècle.

39 Tandis que l'Athos fait l'objet de multiples attentions, au début des années 2000, l'État et l'Église orthodoxe russes mettent en place une collecte de fonds afin de financer les travaux de réfection, d'embellissement et de développement d'infrastructures autour du monastère de Saint Pandeleïmon, dont la première mention en tant qu'établissement des « Russes » daterait de $1016^{88}$.

40 Face à un héritage et une mémoire athonites résolument rattachés à l'Empire byzantin ${ }^{89}$ puis, par association, à la Grèce, les pouvoirs russes cherchent à mettre en exergue une autre mémoire et une autre facette de l'histoire et de la spiritualité athonites : leurs liens anciens et profonds avec la Russie et avec le monde slave.

41 À partir du début des années 2010, sous l'égide du tandem politique Dimitri MedvedevVladimir Poutine et du Patriarcat orthodoxe de Moscou Kyrillos ${ }^{90}$, la commémoration du «millénaire de présence russe sur le Mont Athos» se prépare. Autour de la «Fondation internationale pour la restauration et la conservation du patrimoine culturel et spirituel du monastère Saint-Pandeleïmon ", établie en 2012, une série de colloques consacrés à la " présence russe sur l'Athos » sont programmés dans plusieurs pays d'Europe et en fédération russe ${ }^{91}$; des pèlerinages menés par le haut clergé orthodoxe russe et des visites de fidèles sont organisés ${ }^{92}$. Monastère de SaintPandeleïmon, ermitage du Vieux Roussikon, cellule de Saint Dimitri, etc.: la restauration des établissements "russes" sur l'Athos est érigée en affaire d'État ${ }^{93}$. Après la première visite officielle de Vladimir Poutine en 2005, le 5 juin 2013, le Patriarche de Moscou célèbre la liturgie dans l'église abbatiale restaurée de SaintPandeleïmon ${ }^{94}$. Ces manifestations culminent en 2016. Le programme de commémorations russes est marqué par une visite très médiatisée de Vladimir Poutine 
en Grèce, les 27 et 28 mai 2016. Après un passage à Athènes et la signature d'accords économiques ${ }^{95}$, le Président russe se rend sur l'Athos et, accueilli par le Patriarche de Moscou, la Sainte Communauté et la confrérie de Saint-Pandeleïmon ${ }^{96}$, il souligne la place primordiale du Mont Athos " pour l'histoire de la Russie et pour le peuple russe », son rôle « exceptionnel » aujourd'hui «tandis que la foi orthodoxe s'affermit en Russie et dans d'autres pays » :

Tandis que nous remettons en valeur le patriotisme, la mémoire historique, la culture traditionnelle, nous comptons sur l'affermissement de nos relations avec l'Athos. Il est heureux que d'année en année, de plus en plus de pèlerins russes se rendent ici : ils sont déjà plus de 11000 par an. J'aimerais vous remercier (...) de l'hospitalité qui attend ici nos compatriotes. (...) Les reliques athonites régulièrement exposées en Russie pour y être vénérées sont un témoignage des liens toujours plus étroits entre la Russie et l'Athos. Parmi ces reliques, la Ceinture de la Mère de Dieu apportée du monastère de Vatopédi (...). Des centaines de milliers d'orthodoxes sont venus les vénérer en Russie (...). Nous espérons que cette éminente et noble mission des monastères athonites se poursuivra ${ }^{97}$ ".

Si les journaux européens évoquent alors les « excellentes relations du gouvernement Alexis Tsipras (Syriza, gauche radicale) avec Moscou $»^{98}$, d'autres documents sont moins idylliques.

L'arrivée au pouvoir du Syriza a peut-être insufflé, initialement, un climat plus convivial entre la Grèce et la Russie : les ministères des Affaires étrangères des deux pays ne se seraient-ils pas entendus pour fêter ensemble le millénaire du monachisme russe sur l'Athos ${ }^{99}$ ? Des textes suggèrent pourtant à quel point la politique commémorative russe sur l'Athos a suscité la méfiance de diplomates européens et grecs $^{100}$. Convoquée à ce sujet en 2014 par Aristos Kasmiroglu, le gouverneur civil grec de l'Athos, la Sainte Communauté se serait montrée plutôt "négative » vis-à-vis des festivités mémorielles russes ; notamment les représentants des monastères « grecs (...) subventionnés par l'Union européenne ", qui entretiendraient "des conflits anciens avec les moines russes au sujet des skites et cellules anciennnement russes ${ }^{101}$ ».

$\mathrm{Si}$, pour le quotidien russe Nevazissimaïa Gazeta, les célébrations du $1000^{\mathrm{e}}$ anniversaire $\mathrm{du}$ monastère russe sur l'Athos représenteraient "une nouvelle tentative de démonstration de la grandeur, de l'ancienneté et de l'étendue de l'Église orthodoxe russe à la veille du Grand Concile panorthodoxe ${ }^{102}$ " (prévu le 26 juin de la même année $\left.{ }^{103}\right)$, d'autres commentaires y voient une « humiliation politique pour le Président de la République grecque », Prokopios Pavlopoulos, assis sur un « strapontin » lors de la liturgie, Vladimir Poutine ayant pour sa part investi le trône éspicopal ${ }^{104}$.

À l'été 2018, dans un contexte de tension diplomatique entre la Grèce et la Russie ${ }^{105}$, la presse grecque tend même à dénoncer les «stratégies d'influence géopolitiques » russes en Europe, et notamment en Grèce, par le biais des «structures ecclésiastiques orthodoxes et du Mont Athos » face à l'alliance atlantique et européenne ${ }^{106}$. Est aussi rappelée la rivalité ancienne mais toujours vivace entre le Patriarcat de Moscou et celui de Constantinople, qui possède l'autorité spirituelle sur l'ensemble de l'Athos ${ }^{107}$ : ainsi, début mars 2014, dans une lettre adressée à la Communauté de la Sainte Montagne, le Patriarche grec orthodoxe Bartholomaios aurait-il recommandé de limiter le nombre de moines « non hellénophones » sur l'Athos à $10 \%$ au total ${ }^{108}$.

Si célébrer ensemble un événement peut effectivement contribuer à construire une mémoire partagée et à rapprocher des hommes ou des États, certains épisodes 
historiques, figures, monuments ou territoires peuvent aussi porter des mémoires plurielles, parfois convergentes mais parfois aussi antagoniques ${ }^{109}$.

\section{Conclusion}

Les pratiques patrimoniales et mémorielles autour du Mont Athos depuis la seconde moitié du $\mathrm{xx}^{\mathrm{e}}$ siècle confirment d'abord la dimension de choix et de relecture constante opérée, à partir d'enjeux présents, dans l'écriture et dans la reconstitution d'un passé collectif à un moment donné. Elles permettent aussi de souligner la force mobilisatrice des discours et actions mémoriels, ces «mémoires collectives " posées et revendiquées par un groupe social ou un pouvoir, chargées d'émotion: utilisables comme capital pour un État ou pour une ville dans la formulation de son identité ou le renforcement de son rayonnement international; valorisées comme ressource pour des communautés religieuses attachées à défendre, en se référant au passé, leur statut, des avantages ou une position actuelle; mobilisées comme moyen de légitimer, par l'ancienneté, un ancrage et une influence aujourd'hui et demain sur un territoire. La mise en scène du passé et l'exaltation d'une mémoire communautaire peuvent donc, paradoxalement, représenter des moyens de construire des configurations présentes et futures : ici, le terrain mémoriel, en apparence tourné vers l'histoire, est un « théâtre » où s'expriment et parfois s'affrontent des représentations du passé, reflétant et concrétisant des projets actuels. En ce sens, on peut parler de mémoires performatives ${ }^{110}$ pour évoquer la récente mise en mémoire et en patrimoine du Mont Athos, territoire européen frappé du sceau du sacré. Et, en effet, au-delà même des stratégies mémorielles d'acteurs politiques et au-delà des logiques de sécularisation à l'œuvre dans les actions patrimoniales, l'Athos, ses monastères et ses moines restent enveloppés d'une aura. Cette aura, suscitant émotion, respect, crainte, admiration, est attachée à un îlot spirituel dévoué à Dieu, perçu comme retiré du monde, de son agitation et de ses valeurs dominantes - un espace conçu par beaucoup comme interdit et inviolable.

\section{NOTES}

1. ORWELL George, 1984, Paris, Ebook, 2017 [1948], p. 42.

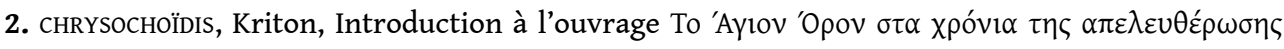

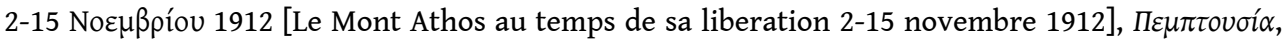
28 avril 2018.

3. Vie commune totale.

4. Quelles que soient leurs origines, les moines orthodoxes acceptés comme permanents par un monastère de l'Athos obtiennent automatiquement la nationalité grecque aujourd'hui. Code de

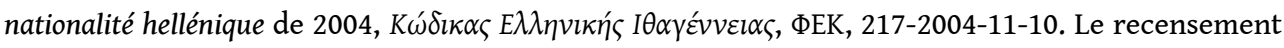
de 2011 comptabilise 1811 moines sur le Mont Athos. 
5. L'État grec, qui exerce un contrôle sur la péninsule (maintien de l'ordre, sécurité publique et militaire), est représenté auprès de la Sainte Communauté par un gouverneur civil relevant du ministère des Affaires étrangères.

6. THOMAS John, CONSTANTINIDIS Angela (dir.), Byzantine Monastic Foundation Documents, Washington,

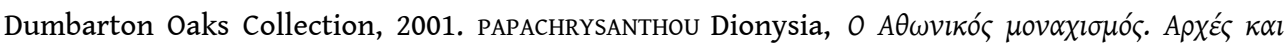
opүóv $\omega \sigma \eta$ [Le monachisme athonite : pouvoirs et organisation], Athènes, Morfotiko Idryma Ethnikis Trapezas, 1992.

7. Charte constitutionnelle du Mont Athos, décret-loi du 10/16 septembre 1926. Article 105 de la Constitution hellénique.

8. JOHAN Michel, Gouverner les mémoires. Les politiques mémorielles en France, Paris, PUF, 2010.

9. Le terme « héritage ", directement repris de l'anglais, est plus souvent traduit en français par "patrimoine» débouchant sur les expressions: "politiques patrimoniales» ou encore "patrimonialisation", NAVARRO Nicolas, «Politiques patrimoniales et touristiques des territoires", Culture et musées $\mathrm{n}^{\circ} 23,2014$, p. 87-107. Je reprends pourtant l'expression "terre d'héritage " à propos du Mont Athos en raison de la dimension sacrée attachée à cet espace ; de la récurrence, à propos du Mont Athos, de la référence à un " héritage transmis par les ancêtres » tant dans les discours des moines que dans celui des historiens, des spécialistes du patrimoine ou encore des politiques.

10. https://whc.unesco.org/en/list/454

11. Une série d'entretiens ont été réalisés en 2016 et 2017 auprès d'acteurs du patrimoine, d'experts, d'archéologues, de prêtres et de moines orthodoxes, de laïcs résidant à Thessalonique et à Ouranopoulis. La publication, depuis les années 1930, d'archives monastiques par des équipes d'historiens a ouvert des sources précieuses permettant de mieux comprendre le fonctionnement des monastères athonites aux époques byzantine et ottomane : Actes de l'Athos, Collège de France,

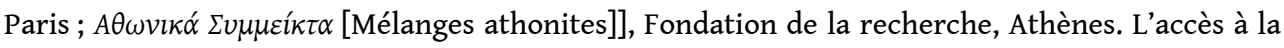
péninsule de l'Athos est rigoureusement interdit aux femmes.

12. La notion même de mémoire collective est controversée car elle impliquerait la création, bien problématique, d'une catégorie holiste en partant de processus relevant de la psychologie individuelle. NOIRIEL Gérard, «Pour une approche subjectiviste du social », Annales ESC 6, 1989, p. 1435-1459.

13. HALBWACHS Maurice, Les cadres sociaux de la mémoire, Paris, Albin Michel, 1925 ; BARLETT Frederic, Remembering. An Experimental and Social Study, Cambridge, Cambridge University Press, 1932 ; PENNBAKER William, PAEZ Dario, RIMÉ Bernard (dir.), Collective Memory of Political Events, Mahwah, Lawrence Erlbaum Associates, 1997.

14. HALBWACHS Maurice, La topographie imaginaire des Évangiles. Étude de mémoire collective, Paris, PUF, 1941.

15. Parmi d'autres, NORA Pierre (dir.), Les Lieux de mémoire, Paris, Gallimard, 3 volumes, 1984, 1986, 1992 ; BASTIDE, Roger, « Mémoire collective et sociologie du bricolage », Année sociologique, vol. 21, 1970, p. 65-108; WERTSCH James, Voices of Collective Remembering, Cambridge, Cambridge University Press, 2002 ; JOUTARD Philippe, La légende des Camisards, Paris, Gallimard, 1977 ; VALENSI Lucette, Les fables de la mémoire. La glorieuse bataille des Trois Rois, Paris, Seuil, 1992 ; NAMER Gérard, Batailles pour la mémoire. La commémoration en France de 1945 à nos jours, Paris, Klinksieck, 1983; VOLLHARDT Johanna Ray, BILEWICz Michael, «After the Genocide. Psychological Perspectives on Victims, Bystanders and Perpetrator Groups ", Journal of Social Issues, vol. 69, $\mathrm{n}^{\circ}$ 1, 2013, p. 1-15.

16. NORA Pierre, «La mémoire collective », dans Jacques Le Goff (dir.), La nouvelle histoire, Paris, Retz-CEPL, 1978, p. 398.

17. En psychologie cognitive, la notion de «métamémoire» désigne, au second degré, les connaissances qu'une personne possède sur la mémoire ou les processus de contrôle de la 
mémoire. CANDAU Joël, «Les processus de la mémoire partagée », Pour, nº 181, 2004, p. 118-123 ; id., Anthropologie de la mémoire, Paris, Armand Colin, 2005, p. 77-78.

18. SEIGNoBos Charles, La méthode historique appliquée aux sciences sociales, Paris, Alcan, 2014 [1901] ; BLOCH Marc, Apologie pour l'histoire ou métier d'historien, Paris, Armand Colin, 1993 [1949].

19. JOUTARD Philippe, Histoire et mémoire. Conflits et alliances, Paris, La Découverte, 2013.

20. NORA Pierre, "Entre histoire et mémoire ", dans Les lieux de mémoire, Paris, Gallimard, vol. 1, 1984, p. XIX; RIMÉ Bernard, CHRISTOPHE Véronique, « How Individual Emotional Episodes Feed Collective Memory », dans James Pennebaker, Dario Paez, Bernard Rimé (dir.), Collective Memory of Political Events. Social Psychological Perspectives, Mahwah, Lawrence Erlbaum Associates, 1997, chapitre 6.

21. KOULOURI Christina (dir.), Clio in the Balkans, Thessaloniki, CDRSEE, 2002, p. 15-48; VALENSI, Les fables de la mémoire, op. cit.

22. RICcEUR Paul, Temps et récit, Paris, Seuil, tome 1, 1983 ; JOUTARD, Histoire et mémoire, op.cit.; AMALvi Christian, «Naissance et affirmation de l'histoire de l'histoire: jalons pour une connaissance de la Nouvelle Clio", dans AMALVI Christian (dir.), Une Passion pour l'histoire. Histoire(s), mémoire(s) et Europe, Paris, Privat, 2002, p. 33-39.

23. RIOUX Jean-Pierre, "Le Tour de la France par deux enfants ", émission La Marche de l'Histoire, France Inter, 26 juin 2012 ; AMALVI Christian, De l'art et la manière d'accommoder les héros de l'histoire de France. De Vercingétorix à la Révolution. Essai de mythologie nationale, Paris, Albin Michel, 1988 ; CITRON Suzanne, Le mythe national. L'histoire de France en question, Paris, Éditions ouvrières, 1987 ; KOULOURI, Clio in the Balkans, op. cit.; ESBENSHADE Richard, «Remembering to Forget. History, National Identity in Post-War East Central Europe », Representations, nº 49, 1995, p. 72-96.

24. LAVABRE Marie-Claire, "Usages du passé, usages de la mémoire", Revue française de science politique, vol. 44, $\mathrm{n}^{\circ} 3,1994$, p. 480-493.

25. NORA, Les lieux de mémoire, op.cit., 1984, 1986, 1992 ; ALCARAZ Emmanuel, Les lieux de mémoire de la guerre d'indépendance algérienne, Paris, Karthala, 2017 ; HARTOG François, REVEL Jacques (dir.), Les usages politiques du passé, Paris, Éditions de l'EHESS, 2001 ; BOUTIER Jean, JULIA Dominique (dir.), Passés recomposés, Champs et chantiers de l'histoire, Paris, Autrement, 1995 ; DIAZ-ANDREU Margarita, CHAMPION Timothy (dir.), Nationalism and Archeology in Europe, Boulder, Westview Press, 1996 ; BOURGUET Marie-Noelle, VAlensi Lucette, wACHTEL Nathan (dir.), Between Memory and History, Londres, Routledge, 2016 ; BOYTNER Roy, SWARTZ DODD Lynn, PARKER Radley (dir.), The Political Uses of Archeology in the Middle East, Tucson, University of Arizona Press, 2010 ; JONEs Sian, The Archeology of Ethnicity. Constructing Identities in the Past and in the Present, Londres, Routledge, 1997; FOWLER Don, «Uses of the Past. Archeology in the Service of the State ", American Antiquity, vol. 52, $\mathrm{n}^{\circ} 2$, 1987, p. 229-248 ; BRow James, "Notes on Community, Hegemony and the Uses of the Past ", Anthropological Quaterly, vol. 63, $\mathrm{n}^{\circ}$ 1, 1980, p. 1-6.

26. RAGARU Nadège, «Usages politiques du passé et controverses historiographiques. Le cas du massacre de Batak », Le Courrier des Pays de l'Est, $n^{\circ}$ 3, 2008, p. 82-87 ; VALTCHINOVA Galia, « Le passé, la nation, la religion. La politique du patrimoine en Bulgarie ", Études balkaniques, $\mathrm{n}^{\circ} 12,2005$, p. 194-209 ; BATUlovic Alenka, « Nationalism in the Classrooms. Narratives of the War in Bosnia Herzegovina (1992-1995) », Study on Ethnicity and Nationalism, vol. 6, n 3, 2006, p. 51-72.

27. BROWN Keith, HAMILAKIS Iannis, The Usable Past. Greek Metahistories, Lanham, Lexington Books, 2003.

28. HAMILAKIS Iannis, The Nation and Its Ruins. Antiquity, Archeology and National Imagination in Greece,

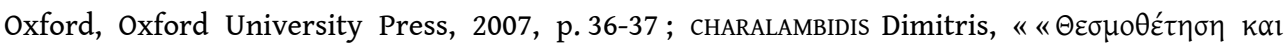

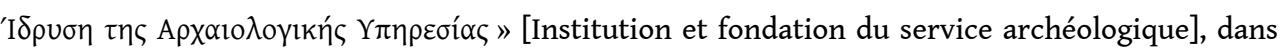

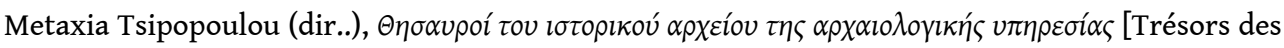


archives historiques du service archéologique], Thessalonique, Ministère de la Culture et du Tourisme/Musée archéologique de Thessalonique, 2010, p. 13-17.

29. Rappelons qu'au $x_{X}{ }^{e}$ siècle, l'héritage culturel, archéologique hellénique n'est pas seulement un enjeu politique interne, mais une affaire européenne. Nombre d'historiens, philologues, archéologues et intellectuels se passionnent pour la Grèce et son histoire, réputée prestigieuse, contribuant aussi, par là, à mettre en valeur et à revisiter cet héritage.

30. RICKS David, MAGDALINo Paul, Byzantium and Modern Greek Identity, Londres, Routledge, 1998 ; DELOUIS Olivier, COUDERC Anne, GURAN Petre (dir.), Héritages de Byzance en Europe du Sud-Est à l'époque contemporaine, Athènes, EFA, 2013.

31. KoUlouRI Christina, "Les manuels scolaires dans l'État grec 1834-1937 », dans Alain Choppin, Manuels scolaires, États et sociétés (XIX ${ }^{e}$-XXe siècles), numéro spécial Histoire de l'éducation, $\mathrm{n}^{\circ}$ 58, 1993, p. 9-26; id., "L'image de l'autre national dans les manuels scolaires grecs au tournant du XIX siècle ", Revue des études sud-est européennes, vol. 33, n ${ }^{\text {os }} 1-2,1995$, p. 15-22 ; KoUMBouRLIS Iannis, La formation de l'histoire nationale grecque. L'apport de Spyridon Zambelios, Athènes, IRN-FNRS, 2005.

32. Hellenic Commission for UNESCO, http://www.unesco-hellas.gr/gr/3_5_1.htm

33. Si le pouvoir politique s'est efforcé, dès 1830-1833, de contrôler l'autorité religieuse, en contrepartie, le christianisme orthodoxe est érigé en composante d'un discours nationaliste, de type conquérant, au $\mathrm{XIX}^{\mathrm{e}}$ siècle et l'institution ecclésiastique est progressivement intégrée à l'appareil d'État. DÉPRET Isabelle, Église orthodoxe et histoire en Grèce contemporaine. Versions officielles et controverses historiographiques, Paris, L'Harmattan, 2009, p. 23-85.

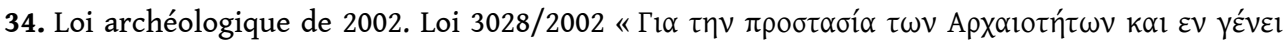

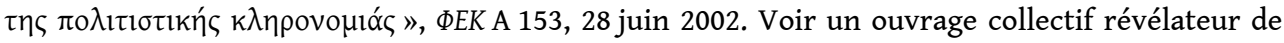
ces évolutions, coordonné par une spécialiste d'architecture byzantine : BROUSKARI Ersi (dir..), $H$

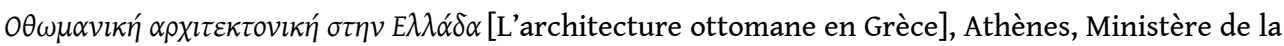
Culture, Direction des Antiquités byzantines et post-byzantines, 2009.

35. Voir le texte très orienté de LAURENT Vittalien, DALLEGGIO Eugenio, «Les études byzantines en Grèce 1940-1948 ", Revue des études byzantines, t. 7, 1949, p. 91-128, qui commence ainsi : «La Grèce est - cela se doit - le pays d'élection des études byzantines ».

36. Organisation des Nations unies pour l'éducation, la science, la culture, Convention concernant la protection du patrimoine mondial culturel et naturel, Paris, Unesco, 1972 : http://whc.unesco.org/ archive/convention

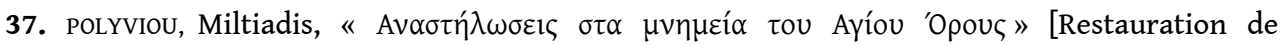

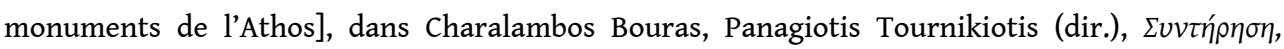

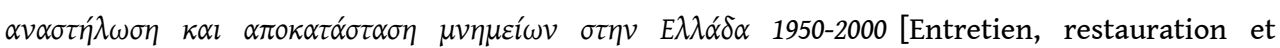
réparation des monuments en Grèce 1950-2000], Athènes, Politistiko Idryma Omilou Peiraios, 2010, p. 199-206 ; Guillou André, BOMPAIRE Jacques, "Recherches au Mont Athos ", Bulletin de correspondance hellénique, vol. 82, 1958, p. 172-192.

38. http://fr.unesco.org/countries/grece/conventions

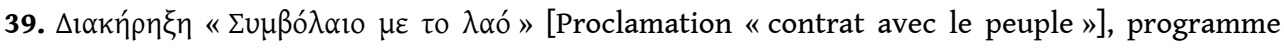
politique du PASOK en vue des législatives d'octobre 1981, Athènes, juillet 1981; PRODROMOU Elizabeth, Democracy, religion and identity in socialist Greece. Church State Relations under PASOK 1981-1989, thèse non publiée, Massachusetts Institute of Technology, Department of Political Sciences, 1993 ; DÉPRET Isabelle, Religion, nation et citoyenneté. L'Église orthodoxe de Grèce et le conflit des cartes d'identité, Paris, L'Harmattan, 2012.

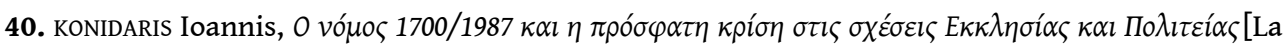
loi 1700/1987 et la récente crise entre l'Église et l'État], Athènes, Sakkoulas, 1991.

41. Nópos 1700-1987 [Textes legislatif 1700-1987, Journal officiel 61/A/6 mai 1987], ФEK 61/A/6 mai 1987 
42. DÉPRET Isabelle, « Tradition orthodoxe et symboles religieux en Grèce. La loi sur le patrimoine ecclésiastique », Archives de sciences sociales des religions, $\mathrm{n}^{\circ}$ 149, 2010, p. 129-150.

43. https://whc.unesco.org/fr/criteres/

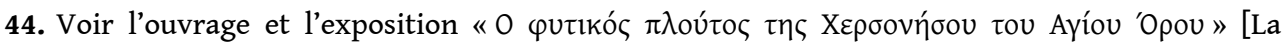
richesse végétale de la péninsule du Mont Athos], préparés par le Centre du Mont Athos (Thessalonique) et les monastères athonites dans le cadre du colloque ECOCITY FORUM 2018 "L'économie cyclique des villes intelligentes » et présentés au Palais de la Musique de Thessalonique, 3-8 octobre 2018.

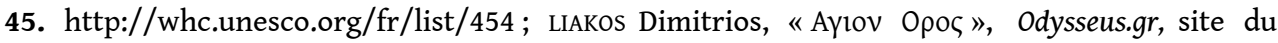
ministère grec de la Culture, http://europa.eu/about-eu/funding-grants/index_fr.htm. En Grèce, http://espa.gr. sMiтH Laurajane, The uses of Heritage, Londres-New York, Routledge, 2006.

46. AHRWEILER Hélène, L'idéologie politique de l'Empire byzantin, Paris, PUF, 1975.

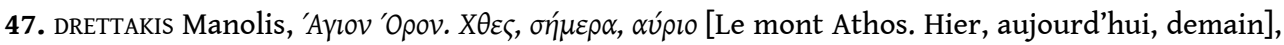
Athènes, En Plo, 2011.

48. JEUDY Jean-Pierre, La machine patrimoniale, Paris, Circé, 2008.

49. Décision ministérielle 5980/16 octobre 1965, ФЕK 714B/29 octobre 1965.

50. Échanges avec le secrétariat général de la Sainte Communauté athonite, juillet, novembre 2015.

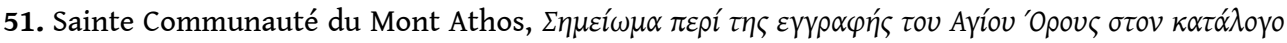
$\pi \varepsilon \rho l o \chi \omega ́ v ~ \mu \nu \eta \mu \varepsilon l \omega ́ v ~ \pi \alpha \gamma \kappa o ́ \sigma \mu l \alpha \varsigma \kappa \lambda \eta \rho o v o \mu l \alpha ́ \varsigma$ [Mémorandum de la Sainte Communauté de la Sainte Montagne d'Athos sur la situation de conservation du Mont Athos dans la perspective de la $32^{\mathrm{e}}$ Assemblée de la Commission du patrimoine mondial], Karyès, 2008.

52. Décision 660/2001 du Conseil juridique de l'État, 24 octobre 2001.

53. Elissaios, higoumène de Simonopetra, «Caractéristiques fondamentales de la société athonite ", allocution au séminaire consacré à la protection du Mont-Athos organisé sous l'égide de l'Unesco, Salonique, 29-30 août 2013.

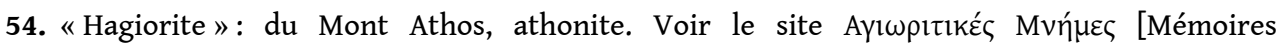
hagiorites]: http://agioritikesmnimes.blogspot.com; Athos memory: http:// athosmemory.blogspot.com/

55. CiTRon Suzanne, "Mémoire nationale, mémoire plurielle ", L'Homme et la société, $\mathrm{n}^{\circ} 1114,1988$,

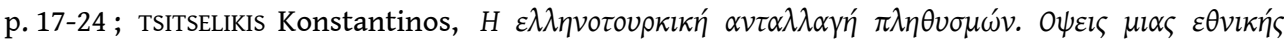

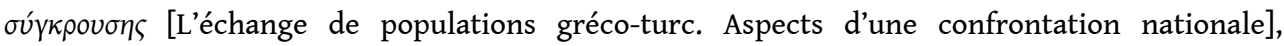
Athènes, KEMO, 2006 ; KARAKASIDOU Anastasia, Fields of Wheat, Hills of Blood: Passages to Nationhood in Greek Macedonia 1870-1990, Chicago, University of Chicago Press, 1997 ; vAN BOESCHOTEN Riki et al.,

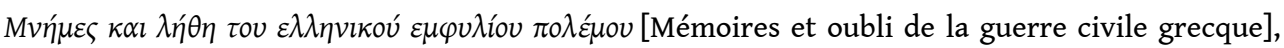
Athènes, Epikentro, 2008.

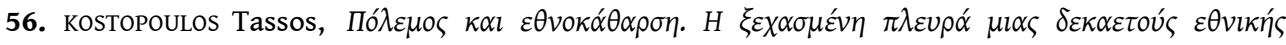
$\varepsilon$ ६óp $\mu \eta \sigma \eta \varsigma$ 1912-1922 [Guerre et nettoyage ethnique. La face oubliée d'une décennie de mobilisation 1912-1922], Athènes, Vivliorama, 2007.

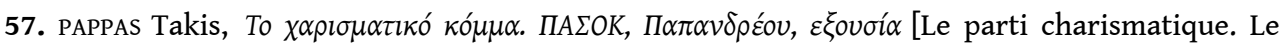
PASOK, Papandreou et le pouvoir], Athènes, Pataki, 2009.

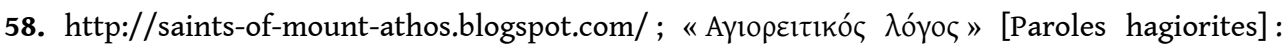
http://www.pemptousia.gr/

59. Les grands monastères de l'Athos se développent aux $\mathrm{x}^{\mathrm{e}}-\mathrm{XI}^{\mathrm{e}}$ siècles; le premier en date, Megisti Lavra, aurait été fondé en 963-964 après J.-C.

60. Centre pour la préservation de l'héritage agiorite, Thessalonique : http://www.kedak.gr/toagion-oros-simera/articles/171/ ; https://athosweblog.com/ ; http://www.pemptousia.gr/ 61. LAURÈs Benjamin, « La vie cénobitique à l'Athos », Échos d'Orient, n 4, 1900, p. 80-87. 
62. VoGüÉ Eugène, Syrie, Palestine, Mont-Athos. Voyages aux pays du passé, Paris, Plon, 1876 ; LACARRIÈre Jacques, Mont-Athos, Montagne sainte, Paris, Seghers, 1954 ; sCHALLER Philippe, "Mont Athos: La Montagne où prient des hommes ", Le Monde, 21 décembre 2010; DARQUES Régis, «Logiques de protection de l'environnement en Grèce. Le cas de la péninsule athonite ", Méditerranée, $\mathrm{n}^{\circ}$ 105, 2005, p. 73-80.

63. DUREL Alain, Parce que tu es tiède. Entretien avec un moine du Mont Athos, Paris, Desclée du Brouwer, 2012.

64. Le monastère comptait 130 moines au début des années 2000, 110 en 2016: https:// www.newsbeast.gr/weekend/arthro/2511883/i-110-monachi-tis-monis-esfigmenou-pou-ine-sana-min-iparchoun,

65. Idem, 22 décembre 2016.

66. Depuis 2005, s'opposent une "ancienne » communauté (zélote) d'Esphigmenou, désavouée par le Patriarcat de Constantinople mais restée indélogée, et une «nouvelle» communauté,

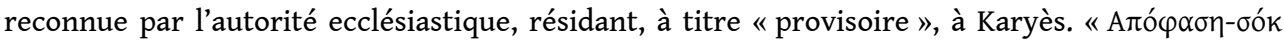

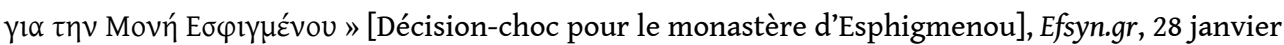
2017.

67. https://www.esphigmenou.gr/

68. BOMPAIRE Jacques, LEFORT Jacques, KRAVARI Vassiliki, GYros Christophe (dir.), Actes de Vatopaidi I, Louvain, Peeters, 2001.

69. Il possède des terres en Macédoine (Pella, Salonique), en Chalcidique, en Thrace et à Athènes.

70. MANDZARIDIS Georges, "New Statistical Data concerning the Monks of Mount Athos ", Social

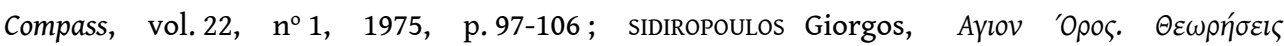
$\alpha \nu \theta \rho \omega \pi о \gamma \varepsilon \omega \gamma \rho \alpha \varphi i \alpha \varsigma$ [Le Mont Athos, considérations de géographie humaine], Athènes, Kastaniotis, 2000.

71. «MOP »/Programmes méditerranéens intégrés, des trois plans-cadres de soutien communautaire/KPS, puis programmes-cadres de référence stratégiques (ESPA). Également Commission de Bruxelles, "Vers une approche intégrée du patrimoine culturel européen ", Bruxelles 22 juillet 2014: http://ec.europa.eu/culture/library/publications/2014-heritagecommunication_fr.pdf. Voir le site http://espa.gr/, qui précise les programmes de "développement et d'investissement», bénéficiant - ou susceptibles de bénéficier - de subventions européennes.

72. Orthodoxie.com, 13 avril 2013.

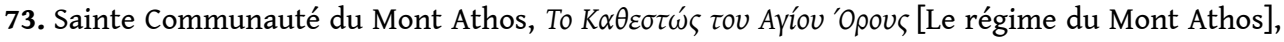
Karyès, Bibliothèque nomocanonique, 1996.

74. ORY Pascal, Une nation pour mémoire: 1889, 1939, 1989, trois jubilés révolutionnaires, Paris, Presses de la Fondation nationale de sciences politiques, 1992.

75. Voir ici BUCUR Maria, Heroes and Victims. Remembering War in Twentieth Century Romania, Bloomington, Indiana University Press, 2009 ; BOTEA Bianca, Territoires en partage. Politiques du passé et expériences de cohabitation en Transylvanie, Paris, Petra, 2013.

76. LAIou Angeliki, «Thessaloniki and Macedonia in the Byzantine period », Byzantine Macedonia, Identity, Image and History, Ancient World Books, Toronto, 2000, p. 1-11; MAZOWER Mark, Salonica, City of Ghosts. Christians, Muslims and Jews, 1430-1950, Londres, Harper Perennial, 2005.

77. DARQUES Régis, Salonique au $\mathrm{XX}^{e}$ siècle. De la Cité ottomane à la métropole grecque, Paris, CNRS, 2000 ; PREVELAKIS Georges, "Salonique entre provincialisme et cosmopolitisme », CEMOTI, $\mathrm{n}^{\circ} 24$, 1997, p. 51-70.

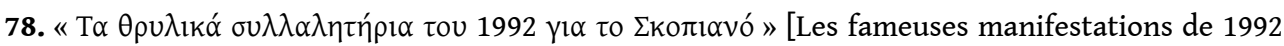

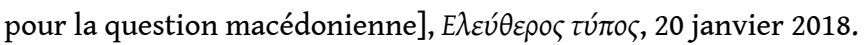

79. https://culture.thessaloniki.gr/afises-arxeia-afison-kith/politistiki-protevousa-tis-evropis/ 
80. PATEL Kiran (dir.), The Cultural Politics of Europe, Londres, Routledge, 2013 ; « European Capitals of Culture»: https://ec.europa.eu/programmes/creative-europe/actions/capitals-culture_en. Dans les années 2010, en pleine crise économique, de nombreux commentateurs dénoncent la mauvaise gestion des fonds de l'organisme «Thessalonique-Capitale européenne de la Culture »

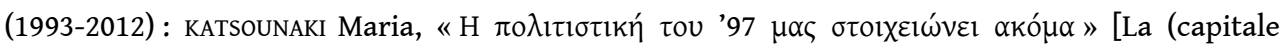

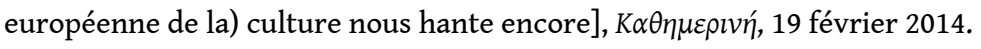

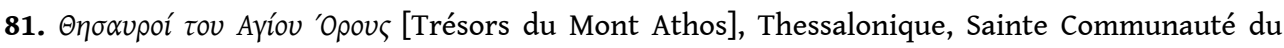
Mont Athos, 1997.

82. Entretiens avec Dimitris Salpistis, commissaire de l'exposition «Les Trésors du Mont Athos », Salonique, 1997. Les monastères de Megisti Lavra, de Kastamonitou, d'Esphigmenou refusent de participer à cette exposition.

83. Ibid.; https://www.agioritikiestia.gr/el/o-foreas/tautotita.

84. À partir des années 2010, sous l'impulsion du maire Ioannis Boutaris, Thessalonique tend à élargir cet héritage mémoriel reconnu et valorisé, en mettant désormais aussi en exergue, malgré des oppositions, le passé multiculturel et multiconfessionnel ottoman de cette ville, les mémoires

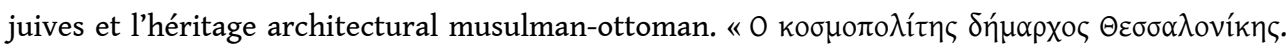

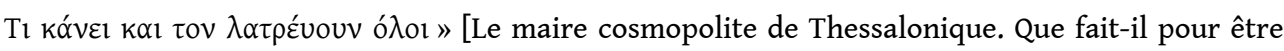
adoré par tous ?], iefimerida.gr, 12 octobre 2012 ; SINTÈs Pierre, GIVRE Olivier, «Iannis Boutaris et les fantômes de Salonique ", Écrire l'histoire, $\mathrm{n}^{\circ}$ 15, 2015, p. 215-222.

85. GERD Lora, Russian Policy in the Orthodox East. The Patriarcate of Constantinople 1878-1914, Berlin, De Gruyter, 2014 ; ROUSSELET Kathy, «La religion au service de l'État russe », Études, mai 2018, p. 83-94; Id., «L'Église russe et la question des frontières. Sainte Russie, monde russe et territoire canonique ", Études du CERI, nº 228-229, 2017, p. 49-53.

86. " Vladimir Poutine célèbre le Noël orthodoxe à Saint Pétersbourg », La croix, 7 janvier 2018.

87. https://www.egliserusse.eu/blogdiscussion/1000-ans-de-presence-russe-au-MontAthos_a1703.html. Selon les données du recensement, en 2006, le monastère comptait environ 50 moines, en majorité d'origine russe.

88. Ibid. Plusieurs milliards de roubles auraient ainsi été récoltés, issus de fidèles et de sponsors.

89. Voir le titre expressif du catalogue de l'exposition " Trésors du Mont Athos », reprise en 2009 à Paris, au Petit Palais : KAKOURIS Isidoros, KOLIOU Madi, CHAZAL Gilles, ZIADÉ Raphaelle (dir.), Le Mont Athos et l'Empire byzantin. Trésors de la Sainte Montagne, Paris, Paris Musées, 2009.

90. LLOBET Anaïs, «Échange de bons procédés entre le Kremlin et l'Église orthodoxe ", Le Monde diplomatique, mars 2018, p. 6-7.

91. Notamment, conférence sur le thème organisée à Belgrade en 2013, à Sofia et à Tcherhiniv (Ukraine) en 2014, à Kiev en 2015, à Thessalonique, à Nicosie et à Moscou en 2016.

92. Église orthodoxe russe, Département des relations internationales : https://mospat.ru/fr/ 2013/04/15/news83549/

93. https://mospat.ru/ru/2014/01/22/news97041/

94. https://mospat.ru/fr/2013/06/05/news86916/

95. « En Grèce, Vladimir Poutine en guerre contre les sanctions de l'UE », Le Monde, 28 mai 2016.

96. « Grèce : Poutine achève sa visite en se rendant sur le Mont Athos », Le Parisien, 28 mai 2016.

97. https://mospat.ru/fr/2016/05/28/news132255/ ; https://orthodoxie.com/le-president-russevladimir-poutine-au-monastere-saint-panteleimon-du-mont-athos/poutin_mibt_athos/ ; «Poutine célèbre la présence russe sur l'Athos », La Croix, 29 mai 2016.

98. https://www.rtbf.be/info/monde/europe/detail_grece-poutine-se-rend-sur-le-mont-athoshaut-lieu-de-la-religion-orthodoxe?id=9310277 ; http://www.efsyn.gr/arthro/live-sto-agionoros-o-poytin

99. https://orthodoxie.com/en/197283-p-2/ 
100. https://orthodoxie.com/les-fonctionnaires-de-l'union-europeenne-par-l'intermédiaire-desautorités-helléniques-tentent-d'exercer-des-pressions/

101. Ibid.

102. Cité par le Courrier international, 30 mai 2016.

103. https://orthodoxie.com/encyclique-du-saint-et-grand-concile-du-27-juin-2016

104. DERVIS-BOURNIAS Dionysios, Huffington Post, 30 mai 2018.

105. «Querelles diplomatiques entre Athènes et Moscou », Le Monde, 11 juillet 2018.

106. "Bouclier anti-missile européen. La Russie se sent visée », Courrier international, 13 mai 2016.

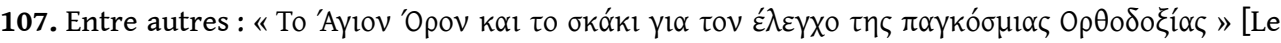
Mont Athos et la lutte pour le contrôle de l'Orthodoxie mondiale], To Bท́ $\mu \alpha, 5$ août 2018.

108. Romfea.gr, 3 mars 2014.

109. RAGARU Nadège, "Commémorer et diviser en Europe. Le $70^{\mathrm{e}}$ anniversaire du sauvetage et des déportations juives depuis les terres sous administration bulgare ", Revue d'études comparatives Est-Ouest, vol. 45, nos 3-4, 2014, p. 237-274.

110. AUSTIN John, How to do things with words, Oxford, Clarendon Press, 1962. Voir l'introduction générale de ce numéro thématique.

\section{RÉSUMÉS}

Haut lieu de la spiritualité chrétienne orthodoxe, le Mont Athos, en tant qu'espace monastique masculin, a traversé les Empires byzantin et ottoman avant d'être intégré à l'État grec entre 1912 et 1926. Depuis les années 1980, le Mont Athos se voit mis en valeur et revisité en "terre d'héritage » et de mémoire, dans un contexte de recomposition de l'Europe. Dans cet article, nous examinons certains des usages du passé, discours et pratiques mémorielles ayant accompagné ces processus récents, en nous interrogeant sur les enjeux et les effets de ces mémoires mobilisées.

As the Mecca of Orthodox Christian spirituality, Mount Athos, a male monastic space, lived through the Byzantine and Ottoman Empires before being integrated into the Greek state between 1912 and 1926. Since the 1980s, Mount Athos has been enhanced and revisited as a "land of heritage" and through memory, in the context of the re-composition of Europe. In this article, we look into some uses of the past, discourses, and practices of memory that have accompanied these recent processes, paying special attention to the stakes and effects of these mobilized memories.

\section{INDEX}

Index géographique : Grèce

Keywords : Greece, Mount Athos, religion and politics, memory practices, memory competitions, uses of the past

Mots-clés : Grèce, Mont Athos, religion et politique, pratiques mémorielles, compétitions mémorielles, usages du passé 
AUTEUR

ISABELLE DÉPRET

Professeur assistante à l'Université de Marmara (Istanbul) et à l'Université libre de Bruxelles isdepret[at]gmail.com 